\title{
SYNCHRONIZED TRAFFIC PLANS AND STABILITY OF OPTIMA
}

\author{
Marc Bernot $^{1}$ And Alessio Figalli ${ }^{2}$
}

\begin{abstract}
The irrigation problem is the problem of finding an efficient way to transport a measure $\mu^{+}$onto a measure $\mu^{-}$. By efficient, we mean that a structure that achieves the transport (which, following [Bernot, Caselles and Morel, Publ. Mat. 49 (2005) 417-451], we call traffic plan) is better if it carries the mass in a grouped way rather than in a separate way. This is formalized by considering costs functionals that favorize this property. The aim of this paper is to introduce a dynamical cost functional on traffic plans that we argue to be more realistic. The existence of minimizers is proved in two ways: in some cases, we can deduce it from a classical semicontinuity argument; the other cases are treated by studying the link between our cost and the one introduced in [Bernot, Caselles and Morel, Publ. Mat. 49 (2005) 417-451]. Finally, we discuss the stability of minimizers with respect to specific variations of the cost functional.
\end{abstract}

Mathematics Subject Classification. 49Q20, 90B10, 90B06, 90 B20.

Received April 20, 2007.

Published online January 30, 2008.

\section{INTRODUCTION}

The variety of structures arising in nature is extraordinary. By exploring the relationship between form and function, D'Arcy Thompson, in his pioneering work [6], tries to find common principles behind the varied phenomena (physical, chemical, biological, short or long time scale, etc.) that interact to give birth to these structures. Indeed, despite the complexity of nature, the approach of retaining only a small but decisive set of parameters and principles to model the phenomenon at the origin of a given structure can be successful. See for example [12] or consider the work of Turing on morphogenesis that led him to explain the appearance of heterogeneous spatial patterns in terms of reaction-diffusion mechanisms [13].

Recently, such an approach was taken to model branched networks that achieve a transport from a source to a target. Such networks are everywhere in nature (plants and trees, river basins, bronchial and cardiovascular systems) and in man designed structures (communication networks, electric power supply, water distribution or drainage networks). The common function of such networks is to transport some goods from an initial distribution (the supply) to another (the demand). Following D'Arcy Thompson, it is desirable to tie a link between this unity of form (branched networks) and this unity of function (transporting goods from a supply to a demand). This was done in $[2,3,5,8,10,15]$ by considering cost functions that encode the efficiency

\footnotetext{
Keywords and phrases. Irrigation problem, traffic plans, dynamical cost, stability.

1 UMPA, ENS Lyon, 46 Allée d'Italie, 69007 Lyon, France; mbernot@umpa.ens-lyon.fr

2 Scuola Normale Superiore, Piazza dei Cavalieri 7, 56100 Pisa, Italy; a.figalli@sns.it
} 
of a transport induced by some structure. Branched structures, as the ones observed in nature, then arise as the optimal structures along which the transport takes place.

A simple but crucial principle was incorporated in the design of all the cost functions used by these authors. This principle states that it is more efficient to transport mass in a grouped way rather than in a separate way. To embed this principle, the previously mentioned costs incorporate a parameter $\alpha \in[0,1]$ and make use of the concavity of $x \mapsto x^{\alpha}$. The idea is that for positive masses $m_{1}$ and $m_{2}$, we have $\left(m_{1}+m_{2}\right)^{\alpha} \leq m_{1}^{\alpha}+m_{2}^{\alpha}$, so that the particles are interested in moving together in order to lower the cost (see for example the role of $\alpha$ in (1.1)). This effect gets stronger as $\alpha$ decreases, while the limit case $\alpha=1$ gives no importance to the grouping of particles.

We now briefly review the different costs and descriptions of branched structures that have been introduced so far. We then introduce a new dynamical cost functional, and enlight the advantages it has over other models.

The model described by Gilbert in [8] consists in finite directed weighed graph $G$ with straight edges $E(G)$ and a weight function $w: E(G) \rightarrow(0, \infty)$. The graph $G$ connects sources $\mu^{+}=\sum_{i=1}^{k} a_{i} \delta_{x_{i}}$ and targets $\mu^{-}=\sum_{j=1}^{l} b_{j} \delta_{y_{j}}$ with $\sum_{i} a_{i}=\sum_{j} b_{j}, a_{i}, b_{j} \geq 0$, and is required to satisfy Kirchhoff's law at each vertex. The cost of $G$ is defined to be:

$$
M^{\alpha}(G)=\sum_{e \in E(G)} w(e)^{\alpha} \mathcal{H}^{1}(e) .
$$

In [15], Xia extends this model to a continuous framework using Radon vector measures. In both these models, the objects and their costs are static in the sense that no "particle" is actually transported along the structure, and the cost depends only on the geometry of the network.

In $[2,3,10]$, a different kind of object, called traffic plan and denoted by $\chi$, is considered. In this framework, all particles are indexed by the set $\Omega:=[0,1]$, and to each $\omega \in \Omega$ is associated a 1-Lipschitz path $\chi(\omega, \cdot)$ in $\mathbb{R}^{N}$. This is a Lagrangian description of the dynamic of particles that can be encoded by the image measure $\mathbf{P}_{\chi}$ of the map $\omega \mapsto \chi(\omega, \cdot)$ (which is therefore a measure on the set of 1-Lipschitz paths). This measure induces a network structure similar to the one considered by Xia. To each traffic plan is associated a cost $E^{\alpha}$ which depends only on its network structure (see Def. 2.4) and, whenever it is finite, is the same as the one considered by Xia. Thus, though a traffic plan is a dynamical object, its cost is static.

In [5], Brancolini, Buttazzo and Santambrogio consider an Eulerian formulation of the problem, describing a transport from $\mu^{+}$to $\mu^{-}$as a path in the space of measures. The cost of such a path is defined as the length induced by a degenerate Riemannian metric in the space of probability measures. More precisely, the cost of a path $\mu(t)$ is given by

$$
\int_{0}^{1} J(\mu(t))\left|\mu^{\prime}\right|(t) \mathrm{d} t
$$

where $J$ is a functional in the space of probability measures and $\left|\mu^{\prime}\right|$ denotes the metric derivative (for the Wasserstein distance) of the path. Both the object and the cost are dynamical in this model.

All the above described models propose structures that transport a measure $\mu^{+}$to a measure $\mu^{-}$and associate a cost to this structure. This leads to consider what is called the irrigation problem by some authors [2-4], i.e., given two measures $\mu^{+}$and $\mu^{-}$, the problem of minimizing the cost among structures transporting $\mu^{+}$to $\mu^{-}$. In the case of the traffic plan model, an additional problem can be considered, namely the who goes where problem $[2,3]$. The latter problem consists in looking for an optimal structure that achieves a given transference plan. In other words, rather than only prescribing the initial an final distribution of masses as in the irrigation problem, one also prescribes the coupling between initial and final positions of each particle. As an example, one can think about the case where the initial distribution represents the habitations, and the final distribution stands for the workplaces. In this case, it is natural to constrain each inhabitant to go from his habitation to his workplace, and so the problem is to find the best itinerary he can follow.

In this paper, we consider the Lagrangian formulation given in [3,10]. This choice is motivated by the fact that traffic plans permit to recover other descriptions. Indeed, given a traffic plan $\chi$, one can always canonically define both a structure similar to the one of Xia, and a path in the space of measures by considering the time 


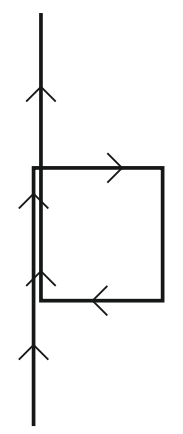

FIGURE 1. In the case of the static cost $E^{\alpha}$ in [3], a portion of a path where it overlaps with itself contributes only once to the total cost, whereas the locality in time of the model we propose gives the expected cost.

marginals of its induced measure $\mathbf{P}_{\chi}$. We consider general costs of the form

$$
\mathcal{C}(\chi):=\int_{\Omega} \int_{\mathbb{R}^{+}} c(\chi, \omega, t)|\dot{\chi}(\omega, t)| \mathrm{d} t \mathrm{~d} \omega .
$$

The advantage of the Lagrangian formulation with respect to the Eulerian one is to allow to define costs of the above form in which one can take care of the speed of each single particle, so that only moving particles contribute to the total cost.

What we propose in this paper, is to give a cost to the actual "dynamical" transport of mass from $\mu^{+}$to $\mu^{-}$that is induced by $\chi$. To obtain such a cost, it is natural to require $c(\chi, \omega, t)$ to be local in space-time. By this property, we mean that $c(\chi, \omega, t)$ only takes into account the particles that are located at the point $\chi(\omega, t)$ at time $t$. In [3] is considered a cost $c(\chi, \omega, t)$ depending on the total mass of particles passing through the point $\chi(\omega, t)$ at some time (see Def. 2.4). Since it takes into account only the global trajectories of particles but not their local dynamics, this cost is local in space but not in time. The associated functional $E^{\alpha}$ thus quantifies the cost of the structure achieving the transport, rather than the cost of the transport itself. In other words, we could also say that $E^{\alpha}$ evaluates the cost of permanent regime connecting $\mu^{+}$to $\mu^{-}$, rather than the cost of a dynamical transport from $\mu^{+}$to $\mu^{-}$. The elementary cost $c$ we introduce in Definition 3.3 has the desired locality property, and we denote by $C^{\alpha}$ the induced cost via formula (1.2). It is possible to extend the time domain by replacing $\mathbb{R}^{+}$with $\mathbb{R}$ in (1.2), and we denote by $E_{\mathbb{R}}^{\alpha}$ and $C_{\mathbb{R}}^{\alpha}$ the costs corresponding to $E^{\alpha}$ and $C^{\alpha}$.

We illustrate the advantage of such a "dynamical" cost with respect to the static one in [3] on two examples:

- It gives a more realistic cost to an overlapping path. Indeed, in the case of the static cost in [3], a path that follows the same circuit twice contributes to the cost once, while the locality in time of the model we propose gives the expected cost (see Fig. 1).

- It is more appropriate for the "who goes where problem". Let us consider the problem of two equal masses $m$ located at points $A$ and $B$, which represent both the source and the target distribution, and where the transference plan constraint consists in switching the two masses. In this case, the solution to this "who goes where" problem is to transport the mass in $A$ to position $B$ and the mass in $B$ to position $A$ along the segment joining them. For such a structure, the $E^{\alpha}$ cost does not distinguish between trajectories going from $A$ to $B$ and from $B$ to $A$. Indeed, the $E^{\alpha}$ cost of this structure is $|A-B|(2 m)^{\alpha}$, while the natural one would be $2|A-B| m^{\alpha}$. This is exactly the cost given by $C^{\alpha}$ (see Fig. 2).

In this paper, we will consider the irrigation problems for all the just mentioned costs. As it will be proved in Section 5, the two irrigation problems with costs $E^{\alpha}$ and $C^{\alpha}$ are equivalent if $\mu^{+}$is a finite atomic, while the equivalence for $E_{\mathbb{R}}^{\alpha}$ and $C_{\mathbb{R}}^{\alpha}$ always holds. More precisely, in these cases, we will prove that any minimizer for the dynamical cost is an $E^{\alpha}$-minimizer, and that moreover, up to reparameterization, the converse is true 


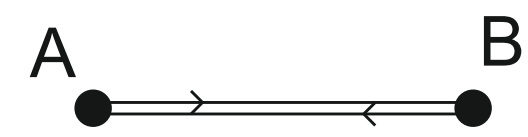

Figure 2. The best way to switch two equal masses between two points $A$ and $B$ is to transport the mass at $A$ to position $B$ and the mass at $B$ to position $A$ along the segment joining them. For such a structure, the $C^{\alpha}$ cost we propose distinguishes between trajectories going from $A$ to $B$ and from $B$ to $A$, which is not the case of the $E^{\alpha}$ cost. Thus, the $C^{\alpha}$ cost is more realistic for the "who goes where" problem.

(see the remarks after Th. 5.2). Since the cost $E^{\alpha}(\chi)$ is invariant by reparameterization of the traffic plan $\chi$, while $C^{\alpha}(\chi)$ in general is not, this fact will tell us in particular that the cost $C^{\alpha}$ has the feature to select, among all the possible reparameterization of an optimal traffic plan $\chi$, some particular ones, in which particles actually move in a grouped way.

Given two measures $\mu^{+}$and $\mu^{-}$, let us define

$$
E^{\alpha}\left(\mu^{+}, \mu^{-}\right):=\inf E^{\alpha}(\chi)
$$

where the infimum is taken over all traffic plans transporting $\mu^{+}$onto $\mu^{-}$(the same can be done with $C^{\alpha}, E_{\mathbb{R}}^{\alpha}$ and $C_{\mathbb{R}}^{\alpha}$ ). By the above formula, one obtains a one-parameter family of distances between measures, each of them inducing the weak-* topology. It turns out that the continuity of the function $\alpha \mapsto E^{\alpha}\left(\mu^{+}, \mu^{-}\right)$is related to the following stability property: given a converging sequence of traffic plans $\chi_{n}$, respectively optimal for the value $\alpha_{n}$, its limit is optimal for the limit value of $\alpha_{n}$. In particular, considering a sequence $\alpha_{n} \rightarrow 1$, one would obtain the convergence of optimal structures to an optimal structure for the 1-Wasserstein distance. It is therefore of interest to study the $\alpha$ dependence of $E^{\alpha}\left(\mu^{+}, \mu^{-}\right)$. This $\alpha$ dependence will be shown in Section 6 to be continuous if $\alpha \in\left[1-\frac{1}{N}, 1\right]$ ( $N$ being the dimension of the ambient space).

The plan is as follows. In Section 2, we recall the principal definitions and results concerning traffic plans. In Section 3, we consider the energy functional of [3] in a more general framework for which we obtain a general lower semicontinuity result. Then we define a new dynamical (in the sense previously discussed) cost functional and obtain a partial result of existence of a "dynamical" optimal traffic plan for the irrigation problem. We can however obtain a more complete existence result by studying the properties of $E^{\alpha}$-minimizers. Indeed, in Section 4 , we prove that any $E^{\alpha}$-optimal traffic plan can be suitably reparameterized. From this fact, we deduce in Section 5 that the cost of optimal traffic plans and dynamical optimal traffic plans are the same, and that any $E^{\alpha}$-optimal traffic plan can be reparameterized so that it is becomes optimal also for the dynamical cost $C^{\alpha}$ (this is always true for $E_{\mathbb{R}}^{\alpha}$ and $C_{\mathbb{R}}^{\alpha}$, while for $E^{\alpha}$ and $C^{\alpha}$ we need $\mu^{+}$to be finite atomic). Finally, in Section 6 , we prove continuity results of $E^{\alpha}\left(\mu^{+}, \mu^{-}\right)$with respect to $\alpha$, for fixed $\mu^{+}$and $\mu^{-}$. As we already said above, this implies that limits of optimal (for different values of $\alpha$ ) traffic plans are still optimal for the limit value.

\section{TRAFFiC PLANS}

In this section, we recall principal definitions and results concerning traffic plans (see $[2-4,10,15]$ ). Let $X$ be some compact convex $N$-dimensional set in $\mathbb{R}^{N}$. We shall denote by $\mathscr{L}^{1}(A)$ the Lebesgue measure of a measurable set $A \subset \mathbb{R}$, and by $\operatorname{Lip}_{1}\left(\mathbb{R}^{+}, X\right)$ the space of 1-Lipschitz curves in $X$ with the metric of uniform convergence on compact sets of $\mathbb{R}^{+}$.

Definition 2.1. Let $\Omega=[0,1]$. A traffic plan is a measurable map $\chi: \Omega \times \mathbb{R}^{+} \rightarrow X$ such that for all $\omega$, $t \mapsto \chi(\omega, t)$ is 1-Lipschitz, and constant for $t$ sufficiently large. Without risk of ambiguity, we shall call fiber both the path $\chi(\omega, \cdot)$ and $\omega \in \Omega$. We denote by $\mathbf{P}_{\chi}$ the law of $\omega \mapsto \chi(\omega) \in \operatorname{Lip}_{1}\left(\mathbb{R}^{+}, X\right)$ defined by $\mathbf{P}_{\chi}(E):=$ $\mathscr{L}^{1}\left(\chi^{-1}(E)\right)$ for every Borel set $E \subset \operatorname{Lip}_{1}\left(\mathbb{R}^{+}, X\right)$.

We remark that in the sequel we will also need to consider the restriction of a traffic plan to a certain subset of fibers $\Omega^{\prime} \subset \Omega$. By abuse of notation, though $\Omega^{\prime}$ will not be of unit mass, we will still call $\chi\left\llcorner\tilde{\Omega}^{\prime}\right.$ a traffic plan. 
Definition 2.2. Two traffic plans $\chi$ and $\chi^{\prime}$ are said to be equivalent if $\mathbf{P}_{\chi}=\mathbf{P}_{\chi^{\prime}}$. In all the following a "traffic plan" means as well the equivalence class of some $\chi$. All proven properties of a traffic plan will be true for any representative up to the addition or removal of a set of fibers with zero measure.

\subsection{Stopping time, irrigated measures, transference plan}

If $\chi: \Omega \times \mathbb{R}^{+} \rightarrow X$ is a traffic plan, define its stopping time by

$$
T_{\chi}(\omega):=\inf \{t \geq 0: \chi(\omega) \text { is constant on }[t, \infty)\} .
$$

Let us denote the initial and final point of a fiber $\omega$ by $\tau(\omega)=\chi(\omega, 0)$ and $\sigma(\omega)=\chi\left(\omega, T_{\chi}(\omega)\right)$. To any $\chi$, one can associate its irrigating and irrigated measure respectively defined by

$$
\begin{gathered}
\mu^{+}(\chi)(A):=\tau_{\#} \mathbf{P}_{\chi}(A)=\mathscr{L}^{1}(\{\omega: \chi(\omega, 0) \in A\}), \\
\mu^{-}(\chi)(A):=\sigma_{\#} \mathbf{P}_{\chi}(A)=\mathscr{L}^{1}\left(\left\{\omega: \chi\left(\omega, T_{\chi}(\omega)\right) \in A\right\}\right),
\end{gathered}
$$

where $A$ is any Borel subset of $\mathbb{R}^{N}$.

\subsection{Energy of a traffic plan}

Definition 2.3. Let $\chi: \Omega \times \mathbb{R}^{+} \rightarrow X$ be a traffic plan. Define the path class of $x \in \mathbb{R}^{N}$ in $\chi$ as the set

$$
\Omega_{x}^{\chi}:=\{\omega: x \in \chi(\omega, \mathbb{R})\},
$$

and the multiplicity of $\chi$ at $x$ by $\theta_{\chi}(x)=\mathscr{L}^{1}\left(\Omega_{x}^{\chi}\right)$. For simplicity, we shall write $\Omega_{x}:=\Omega_{x}^{\chi}$, whenever the underlying traffic plan $\chi$ is not ambiguous.

We use the convention that $0^{\alpha-1}=+\infty$ for $\alpha \in[0,1)$.

Definition 2.4. Let $\alpha \in[0,1]$. We call energy of a traffic plan $\chi: \Omega \times \mathbb{R}^{+} \rightarrow X$ the functional

$$
E^{\alpha}(\chi)=\int_{\Omega} \int_{\mathbb{R}^{+}} \theta_{\chi}(\chi(\omega, t))^{\alpha-1}|\dot{\chi}(\omega, t)| \mathrm{d} t \mathrm{~d} \omega .
$$

Let $\mu^{+}, \mu^{-}$be two probability measures in $X$. Denote by $\operatorname{TP}\left(\mu^{+}, \mu^{-}\right)$the set of traffic plans $\chi$ such that $\mu^{+}(\chi)=\mu^{+}$and $\mu^{-}(\chi)=\mu^{-}$. If $C>0$, call $\mathrm{TP}_{C}$ the set of traffic plans such that $\int_{\Omega} T_{\chi}(\omega) \mathrm{d} \omega \leq C$ and $\operatorname{TP}_{C}\left(\mu^{+}, \mu^{-}\right):=\operatorname{TP}\left(\mu^{+}, \mu^{-}\right) \cap \operatorname{TP}_{C}$.

\subsection{Convergence}

Definition 2.5. We say that a sequence of traffic plans $\chi_{n}$ converges to a traffic plan $\chi$ if $\mathbf{P}_{\chi_{\mathbf{n}}}$ weakly-* converges to $\mathbf{P}_{\chi}$, or equivalently if the random variables $\chi_{n}$ converge in law to $\chi$.

Definition 2.6. We say that a sequence of traffic plans $\chi_{n}$ fiber converges to a traffic plan $\chi$ if $\chi_{n}(\omega)$ converges to $\chi(\omega)$ uniformly on compact subsets of $\mathbb{R}^{+}$for every $\omega \in \Omega$.

Remark 2.7. By Skorokhod theorem (see Th. 11.7.2 [7]) $\chi_{n}$ converges to $\chi$ if and only if there exist $\tilde{\chi}_{n}$ and $\tilde{\chi}$ equivalent to $\chi_{n}$ and $\chi$ respectively and such that $\tilde{\chi}_{n}$ fiber converges to $\tilde{\chi}$.

Proposition 2.8. Up to a subsequence, any sequence of traffic plans $\chi_{n}$ in $\mathrm{TP}_{C}$ converges to a traffic plan $\chi$. In addition, $\mu^{+}\left(\chi_{n}\right) \rightarrow \mu^{+}(\chi)$ and $\mu^{-}\left(\chi_{n}\right) \rightarrow \mu^{-}(\chi)$. 


\subsection{Existence of minimizers}

The optimization problem we are interested in is the irrigation problem, i.e. the problem of minimizing $E^{\alpha}(\chi)$ in $\operatorname{TP}\left(\mu^{+}, \mu^{-}\right)$. The following results are proved in $[2,3,10]$.

Theorem 2.9. If $C>0$ and $\chi_{n}: \Omega \times \mathbb{R}^{+} \rightarrow X$ is a sequence in $\mathrm{TP}_{C}$ converging to the traffic plan $\chi$, then

$$
E^{\alpha}(\chi) \leq \liminf _{n} E^{\alpha}\left(\chi_{n}\right)
$$

We notice that the $\operatorname{cost} E^{\alpha}(\chi)$ is invariant by time-reparameterization of $\chi$. Therefore, one can always reparameterize $\chi$ so that $|\dot{\chi}(\omega, t)|=1$ for all $t \in\left(0, T_{\chi}(\omega)\right)$ without changing the cost. In this case, since $\theta_{\chi}^{\alpha-1} \geq 1$, one gets $\int_{\Omega} T_{\chi}(\omega) \mathrm{d} \omega \leq E^{\alpha}(\chi)$. Thus, if $\chi_{n}$ is a sequence of traffic plan with a uniformly bounded $E^{\alpha}$ cost, it is in $\operatorname{TP}_{C}$ up to reparameterization for $C$ big enough. By Proposition 2.8 and Theorem 2.9, the direct method of the calculus of variations ensures the existence of an optimal traffic plan in $\operatorname{TP}\left(\mu^{+}, \mu^{-}\right)$.

Corollary 2.10. The problem of minimizing $E^{\alpha}(\chi)$ in $\operatorname{TP}\left(\mu^{+}, \mu^{-}\right)$admits a solution.

Definition 2.11. A traffic plan $\chi$ is said to be optimal for the irrigation problem if it is of minimal cost in $\operatorname{TP}\left(\mu^{+}(\chi), \mu^{-}(\chi)\right)$.

Let

$$
E^{\alpha}\left(\mu^{+}, \mu^{-}\right):=\min _{\operatorname{TP}\left(\mu^{+}, \mu^{-}\right)} E^{\alpha}(\chi) .
$$

As proved in [3], there is an optimal traffic plan in $\operatorname{TP}\left(\mu^{+}, \mu^{-}\right)$which is loop-free, i.e. for almost any $\omega \in \Omega$, the map $\chi(\omega, \cdot)$ is one to one in $\left[0, T_{\chi}(\omega)\right]$. Moreover, using Propositions 6.4 and 6.6 in [3], given any optimal traffic plan with finite energy there is an equivalent loop-free traffic plan with the same energy, hence optimal. Thus, without loss of generality, we may assume that optimal traffic plans are loop-free.

The triangle inequality for the cost $E^{\alpha}$ holds (just think of concatenating traffic plans [4]):

Proposition 2.12. Let $\mu_{0}, \mu_{1}$ and $\mu_{2}$ be probability measures. We have the triangle inequality

$$
E^{\alpha}\left(\mu_{0}, \mu_{2}\right) \leq E^{\alpha}\left(\mu_{0}, \mu_{1}\right)+E^{\alpha}\left(\mu_{1}, \mu_{2}\right) .
$$

\subsection{Stability with respect to $\mu^{+}$and $\mu^{-}$}

The following results were first proved in a slightly different framework by Xia [15], and their proofs adapt immediately to traffic plans (see [2]). We remark that, here and in the sequel, by atomic measure we mean a finite sum of delta measures.

Let $C$ be a cube with edge length $L$ and center $c$. Let $\nu$ be a probability measure on the compact set $X$ where $X \subset C$. We may approximate $\nu$ by atomic measures as follow. For each $i$, let

$$
\mathcal{C}_{i}:=\left\{C_{i}^{h}: h \in \mathbb{Z}^{N} \cap\left[0,2^{i}\right)^{N}\right\}
$$

be a partition of $C$ into cubes of edge length $\frac{L}{2^{i}}$. Now, for each $h \in \mathbb{Z}^{N} \cap\left[0,2^{i}\right)^{N}$, let $c_{i}^{h}$ be the center of $C_{i}^{h}$ and $m_{i}^{h}=\nu\left(C_{i}^{h}\right)$ be the $\nu$ mass of the cube $C_{i}^{h}$.

Definition 2.13. We define the dyadic approximation of $\nu$ as

$$
A_{i}(\nu):=\sum_{h \in \mathbb{Z}^{N} \cap\left[0,2^{i}\right)^{N}} m_{i}^{h} \delta_{c_{i}^{h}} .
$$

We observe that the measures $A_{i}(\nu)$ weakly-* converge to $\nu$. 
Proposition 2.14. Let $\alpha \in\left(1-\frac{1}{N}, 1\right]$. Let $\nu$ be a probability measure with support in a cube centered at $c$ and of edge length $L$. We have

$$
E^{\alpha}\left(A_{n}(\nu), \nu\right) \leq \frac{2^{n(N(1-\alpha)-1)}}{2^{1-N(1-\alpha)}-1} \frac{\sqrt{N} L}{2}
$$

In particular, $E^{\alpha}\left(A_{n}(\nu), \nu\right) \rightarrow 0$ locally uniformly in $\alpha$ for all $\nu$ when $n \rightarrow \infty$.

By this result and Theorem 2.9, it is not difficult to prove that the cost $E^{\alpha}$ metrizes the weak-* convergence for $\alpha \in\left(1-\frac{1}{N}, 1\right]$.

Lemma 2.15. Let $\alpha \in\left(1-\frac{1}{N}, 1\right]$. A sequence of probability measures $\nu_{n}$ weakly-* converges to $\nu$ if and only if $E^{\alpha}\left(\nu_{n}, \nu\right) \rightarrow 0$ when $n \rightarrow \infty$.

Corollary 2.16. Let $\alpha \in\left(1-\frac{1}{N}, 1\right]$. If $\chi_{n}$ is a sequence of optimal traffic plans for the irrigation problem and $\chi_{n} \rightarrow \chi$, then $\chi$ is optimal.

Moreover, by Proposition 2.14, $E^{\alpha}\left(\mu^{+}, \mu^{-}\right)$is always finite for $\alpha \in\left(1-\frac{1}{N}, 1\right]$.

\subsection{Regularity}

The following regularity results were proved in [4].

Proposition 2.17. Let $\mu^{+}$and $\mu^{-}$be atomic probability measures and $\alpha \in[0,1]$. An optimum for the irrigation problem is a finite tree made of segments (in the sense that the fibers $\chi(\omega, \cdot)$, once parameterized by arc lengths, describe a finite set of piecewise linear curves).

Theorem 2.18. Let $\alpha \in\left(1-\frac{1}{N}, 1\right)$ and let $\chi$ be an optimal traffic plan in $\operatorname{TP}\left(\mu^{+}, \mu^{-}\right)$. Assume that the supports of $\mu^{+}$and $\mu^{-}$are at positive distance. In any closed ball $B(x, r)$ not meeting the supports of $\mu^{+}$and $\mu^{-}$, the traffic plan has the structure of a finite graph.

\subsection{Extension of the time domain}

In Sections 4 and 5, we will consider traffic plans defined on $\Omega \times \mathbb{R}$. All the notions introduced above are easy to generalize, and we shall denote by $\operatorname{TP}_{\mathbb{R}}\left(\mu^{+}, \mu^{-}\right)$the set of extended traffic plans from $\mu^{+}$and $\mu^{-}$and $E_{\mathbb{R}}^{\alpha}$ the corresponding cost. We denote by $\operatorname{TP}_{\mathbb{R}, C}\left(\mu^{+}, \mu^{-}\right)$the traffic plans $\chi \in \operatorname{TP}_{\mathbb{R}}\left(\mu^{+}, \mu^{-}\right)$such that $\int_{\Omega} T_{\chi}(\omega) \mathrm{d} \omega \leq C$, where for a traffic plan in $\mathrm{TP}_{\mathbb{R}}$

$$
T_{\chi}(\omega):=\inf \{t+s: t, s \geq 0, \chi(\omega) \text { is constant on }(-\infty,-s] \cup[t, \infty)\} .
$$

Any traffic plan $\chi \in \mathrm{TP}_{\mathbb{R}}$ can be shifted in time so that it can be seen as a traffic plan in $\mathrm{TP}$ and the corresponding $E_{\mathbb{R}}^{\alpha}$ and $E^{\alpha}$ costs are the same. Thus, from the point of view of the irrigation problem, the two formalisms yield the same optimal objects. However, the introduction of this extended model is made necessary for the study of the dynamical framework we propose, since the dynamical cost we will consider is not invariant by time-reparameterization.

\section{Dynamic Cost of a tRAFFiC Plan}

Let $\chi$ be a traffic plan and $c(\chi, \omega, t)$ the elementary cost due to the particle $\omega$ along the fiber $\chi(\omega)$ at time $t$. We define a general cost function $\mathcal{C}$ of a traffic plan $\chi$ as follows:

$$
\mathcal{C}(\chi):=\int_{\Omega} \int_{\mathbb{R}^{+}} c(\chi, \omega, t)|\dot{\chi}(\omega, t)| \mathrm{d} t \mathrm{~d} \omega .
$$


The choice $c(\chi, \omega, t)=\theta_{\chi}(\chi(\omega, t))^{\alpha-1}$ yields the energy of a traffic plan given by Definition 2.4. In this section, we first prove that for a large class of elementary $\operatorname{costs} c(\chi, \omega, t)$, the cost of a traffic plan $\mathcal{C}(\chi)$ is lower semicontinuous. Then, we introduce a dynamical elementary cost (see the introduction for the meaning of dynamical) for which the corresponding $\operatorname{cost} \mathcal{C}$ is lower semicontinuous. This yields the existence of a minimizer for the dynamical irrigation problem.

Proposition 3.1. Let $c: \operatorname{TP}\left(\mu^{+}, \mu^{-}\right) \times \Omega \times \mathbb{R}^{+} \rightarrow \mathbb{R}^{+}$such that $c(\cdot, \omega, \cdot)$ is lower semicontinuous (with respect to the fiber convergence on traffic plans and the usual topology in $\mathbb{R}^{+}$) for all $\omega$. If $\chi_{n}: \Omega \times \mathbb{R}^{+} \rightarrow X$ fiber converges to the traffic plan $\chi$, then

$$
\mathcal{C}(\chi) \leq \liminf _{n} \mathcal{C}\left(\chi_{n}\right)
$$

Proof. Let us set $c^{\lambda}(\chi, \omega, t):=\inf _{s \geq 0}\{c(\chi, \omega, s)+\lambda|t-s|\}$. Since $c(\chi, \omega, \cdot)$ is lower semicontinuous, it is classical (see [1]) that $c^{\lambda}(\chi, \omega, \cdot)$ is $\lambda$-Lipschitz and that

$$
c(\chi, \omega, t)=\sup _{\lambda} c^{\lambda}(\chi, \omega, t) .
$$

Let us prove that, $c^{\lambda}(\cdot, \omega, t)$ is lower semicontinuous for all $\omega$ and $t$. Let $\chi_{n} \rightarrow \chi$, and, for fixed $\omega$ and $t$, assume that up to a subsequence the $\lim \inf$ of $c^{\lambda}\left(\chi_{n}, \omega, t\right)$ is indeed a limit. Now, for each $n$, take $t_{n}$ such that

$$
c^{\lambda}\left(\chi_{n}, \omega, t\right) \geq c\left(\chi_{n}, \omega, t_{n}\right)+\lambda\left|t-t_{n}\right|-\frac{1}{n} .
$$

If $t_{n} \rightarrow+\infty$, since $c$ is non-negative,

$$
\lim _{n} c^{\lambda}\left(\chi_{n}, \omega, t\right) \geq \liminf _{n} \lambda\left|t-t_{n}\right|=+\infty \geq c^{\lambda}(\chi, \omega, t) .
$$

Otherwise, up to a subsequence, we can assume $t_{n} \rightarrow t_{\infty}$, so that $\liminf _{n} c\left(\chi_{n}, \omega, t_{n}\right) \geq c\left(\chi, \omega, t_{\infty}\right)$. Therefore,

$$
\lim _{n} c^{\lambda}\left(\chi_{n}, \omega, t\right) \geq \liminf _{n} c\left(\chi_{n}, \omega, t_{n}\right)+\lambda\left|t-t_{n}\right| \geq c\left(\chi, \omega, t_{\infty}\right)+\lambda\left|t-t_{\infty}\right| \geq c^{\lambda}(\chi, \omega, t) .
$$

Let us fix now $T>0$ and $\varepsilon>0$, and let us consider $0=t_{1} \leq \ldots \leq t_{i} \leq \ldots \leq t_{k}=T$ such that $\left|t_{i+1}-t_{i}\right| \leq \varepsilon$. Since $c^{\lambda}(\chi, \omega, \cdot)$ is $\lambda$-Lipschitz, $|\chi(\omega, \cdot)|$ is 1-Lipschitz, and $\chi \mapsto \int|\dot{\chi}(\omega, t)| \mathrm{d} t$ and $\chi \mapsto c^{\lambda}(\chi, \omega, t)$ are lower semicontinuous for the fiber convergence, we have:

$$
\begin{aligned}
\liminf _{n} \int_{[0, T]} c^{\lambda}\left(\chi_{n}, \omega, t\right)\left|\dot{\chi}_{n}(\omega, t)\right| \mathrm{d} t & \geq \sum_{i}\left[\liminf _{n} c^{\lambda}\left(\chi_{n}, \omega, t_{i}\right) \int_{t_{i}}^{t_{i+1}}\left|\dot{\chi}_{n}(\omega, t)\right| \mathrm{d} t-\lambda \varepsilon\left(t_{i+1}-t_{i}\right)\right] \\
& \geq \sum_{i}\left[c^{\lambda}\left(\chi, \omega, t_{i}\right) \int_{t_{i}}^{t_{i+1}}|\dot{\chi}(\omega, t)| \mathrm{d} t-\lambda \varepsilon\left(t_{i+1}-t_{i}\right)\right] \\
& \geq \int_{[0, T]} c^{\lambda}(\chi, \omega, t)|\dot{\chi}(\omega, t)| \mathrm{d} t-2 \lambda \varepsilon T .
\end{aligned}
$$

This being true for all $\varepsilon$, we get for a.e. $\omega$ and all $T>0$,

$$
\begin{aligned}
\liminf _{n} \int_{\mathbb{R}^{+}} c\left(\chi_{n}, \omega, t\right)\left|\dot{\chi}_{n}(\omega, t)\right| \mathrm{d} t & \geq \liminf _{n} \int_{[0, T]} c\left(\chi_{n}, \omega, t\right)\left|\dot{\chi}_{n}(\omega, t)\right| \mathrm{d} t \\
& \geq \liminf _{n} \int_{[0, T]} c^{\lambda}\left(\chi_{n}, \omega, t\right)\left|\dot{\chi}_{n}(\omega, t)\right| \mathrm{d} t \geq \int_{[0, T]} c^{\lambda}(\chi, \omega, t)|\dot{\chi}(\omega, t)| \mathrm{d} t .
\end{aligned}
$$


Then, by Fatou's lemma,

$$
\begin{aligned}
\liminf _{n} \mathcal{C}\left(\chi_{n}\right) & =\liminf _{n} \int_{\Omega} \int_{\mathbb{R}^{+}} c\left(\chi_{n}, \omega, t\right)\left|\dot{\chi}_{n}(\omega, t)\right| \mathrm{d} t \mathrm{~d} \omega \\
& \geq \int_{\Omega} \liminf _{n} \int_{\mathbb{R}^{+}} c\left(\chi_{n}, \omega, t\right)\left|\dot{\chi}_{n}(\omega, t)\right| \mathrm{d} t \mathrm{~d} \omega \geq \int_{\Omega} \int_{[0, T]} c^{\lambda}(\chi, \omega, t)|\dot{\chi}(\omega, t)| \mathrm{d} t \mathrm{~d} \omega,
\end{aligned}
$$

and we conclude thanks to the monotone convergence theorem.

We now define the dynamical multiplicity of $\omega$ at time $t$ as the proportion of particles that are exactly at the same place as $\omega$ at time $t$.

Definition 3.2. Let $\chi: \Omega \times \mathbb{R}^{+} \rightarrow X$ be a traffic plan. We define the path class of $(\omega, t) \in \Omega \times \mathbb{R}$ in $\chi$ as the set

$$
[\omega, t]_{\chi}:=\left\{\omega^{\prime}: \chi\left(\omega^{\prime}, t\right)=\chi(\omega, t)\right\}
$$

and the multiplicity of $\chi$ at $(\omega, t)$ by $\tilde{\theta}_{\chi}(\omega, t):=\mathscr{L}^{1}\left([\omega, t]_{\chi}\right)$.

Definition 3.3. Let $\alpha \in[0,1]$. We call dynamical cost of a traffic plan $\chi: \Omega \times \mathbb{R}^{+} \rightarrow X$ the functional

$$
C^{\alpha}(\chi)=\int_{\Omega} \int_{\mathbb{R}^{+}} \tilde{\theta}_{\chi}(\omega, t)^{\alpha-1}|\dot{\chi}(\omega, t)| \mathrm{d} t \mathrm{~d} \omega
$$

i.e. $C^{\alpha}(\chi)=\mathcal{C}(\chi)$ with $c(\chi, t, \omega)=\tilde{\theta}_{\chi}(\omega, t)^{\alpha-1}$.

Theorem 3.4. If $\chi_{n}: \Omega \times \mathbb{R}^{+} \rightarrow X$ is a sequence in $\operatorname{TP}\left(\mu^{+}, \mu^{-}\right)$converging to the traffic plan $\chi$, then

$$
C^{\alpha}(\chi) \leq \liminf _{n} C^{\alpha}\left(\chi_{n}\right)
$$

Proof. Let us denote

Setting

$$
\delta(x, y)= \begin{cases}0 & \text { if } x \neq y \\ 1 & \text { if } x=y\end{cases}
$$

$$
c(\chi, \omega, t):=\left[\int_{\Omega} \delta\left(\chi(\omega, t), \chi\left(\omega^{\prime}, t\right)\right) \mathrm{d} \omega^{\prime}\right]^{\alpha-1}
$$

where $\alpha \in[0,1]$, we observe that $c(\chi, t, \omega)=\tilde{\theta}_{\chi}(\omega, t)^{\alpha-1}$, so that $C^{\alpha}(\chi)=\mathcal{C}(\chi)$ as defined by (3.1). Let us consider a sequence of traffic plans $\chi_{n}$ fiber converging to $\chi$, and $t_{n} \rightarrow t$. We remark that the function

$$
\mathbb{R}^{N} \times \mathbb{R}^{N} \ni(x, y) \mapsto \delta(x, y) \in \mathbb{R}
$$

is upper semicontinuous. Therefore, since $\chi_{n}(\omega)$ is a 1-Lipschitz curve, if $\chi_{n}(\omega) \rightarrow \chi(\omega)$ and $t_{n} \rightarrow t$ we have

$$
\limsup _{n} \delta\left(\chi_{n}\left(\omega, t_{n}\right), \chi_{n}\left(\omega^{\prime}, t_{n}\right)\right) \leq \delta\left(\chi(\omega, t), \chi\left(\omega^{\prime}, t\right)\right) .
$$

Thus, by Fatou's lemma,

$$
\underset{n}{\limsup } \int_{\Omega} \delta\left(\chi_{n}\left(\omega, t_{n}\right), \chi_{n}\left(\omega^{\prime}, t_{n}\right)\right) \mathrm{d} \omega^{\prime} \leq \int_{\Omega} \delta\left(\chi(\omega, t), \chi\left(\omega^{\prime}, t\right)\right) \mathrm{d} \omega^{\prime}
$$

and since $\alpha \leq 1$,

$$
\liminf _{n} c\left(\chi_{n}, \omega, t_{n}\right) \geq c(\chi, \omega, t) .
$$

Therefore Proposition 3.1 ensures that $C^{\alpha}$ is lower semicontinuous. 
Remark 3.5. It is not difficult to prove the upper semicontinuity of the multiplicity $\theta_{\chi}(\chi(\omega, t))$, so that the elementary cost $c(\chi, \omega, t)=\theta_{\chi}(\chi(\omega, t))^{\alpha-1}$ satisfies the hypothesis of Proposition 3.1. This yields a new simple proof of Theorem 2.9 .

Like in the last paragraph of Section 2, it is possible to consider a dynamical cost $C_{\mathbb{R}}^{\alpha}(\chi)$ for $\chi \in \mathrm{TP}_{\mathbb{R}}\left(\mu^{+}, \mu^{-}\right)$. Proposition 3.1 and Theorem 3.4 hold with $\mathrm{TP}_{\mathbb{R}}$ and $C_{\mathbb{R}}^{\alpha}$ in place of $\mathrm{TP}$ and $C^{\alpha}$. The compactness of $\mathrm{TP}_{C}$ stated in Proposition 2.8 yields:

Proposition 3.6. Let $\mu^{+}$and $\mu^{-}$be probability measures on $X$, and let $C>0$ be such that $\operatorname{TP}_{C}\left(\mu^{+}, \mu^{-}\right)$is not empty (for example, take $C \geq \operatorname{diam}(X)$ ). Then, there exist $C^{\alpha}$-minimizers (resp. $C_{\mathbb{R}}^{\alpha}$-minimizers) in $\mathrm{TP}_{C}$ (resp. $\left.\mathrm{TP}_{\mathbb{R}, C}\right)$.

The argument used to prove Corollary 2.10 (that states the existence of $E^{\alpha}$-minimizers in TP) is not adaptable to the case of $C^{\alpha}$, since neither $C^{\alpha}(\chi)$ nor $C_{\mathbb{R}}^{\alpha}(\chi)$ are invariant by time-reparameterization of $\chi$. In particular, the situation where $C_{\alpha}$-minimizers in $\mathrm{TP}_{C}$ change as $C$ increases to $+\infty$ is not excluded (this is not the case for $E^{\alpha}$, since by the reparameterization argument used to prove Cor. 2.10 we know that all minimizers are in $\mathrm{TP}_{C}$ for $C=E^{\alpha}\left(\mu^{+}, \mu^{-}\right)$). However, we shall see in Section 5 , that by using synchronization techniques developed in Section 4 we are still able to prove existence of $C^{\alpha}$-minimizers in $\operatorname{TP}\left(\mu^{+}, \mu^{-}\right)$provided that $\mu^{+}$is finite atomic, and of $C_{\mathbb{R}}^{\alpha}$-minimizers in $\operatorname{TP}_{\mathbb{R}}\left(\mu^{+}, \mu^{-}\right)$.

\section{Synchronizable trafFic Plans}

Let us define the support of a traffic plan $\chi$ as the set of points with positive multiplicity. This set will be denoted by $S_{\chi}$.

Definition 4.1. A traffic plan $\chi \in \operatorname{TP}_{\mathbb{R}}\left(\mu^{+}, \mu^{-}\right)$(resp. $\operatorname{TP}\left(\mu^{+}, \mu^{-}\right)$) is said to be synchronized (resp. positive synchronized) if it is loop-free, and for all $x$ in the support of $\chi$ there is a time $t_{\chi}(x)$ such that $\chi\left(\omega, t_{\chi}(x)\right)=x$ for all $\omega \in \Omega_{x}$ (i.e. all fibers which pass through $x$ have to pass at the same time).

Given two traffic plans $\chi$ and $\tilde{\chi}$, we say that $\tilde{\chi}$ is a reparameterization of $\chi$ if, for almost every $\omega \in \Omega$, the curve $\tilde{\chi}(\omega, \cdot)$ is a reparameterization of $\chi(\omega, \cdot)$. We will say that $\tilde{\chi}$ is an arc length parameterization of $\chi$ if, for almost every $\omega, \tilde{\chi}(\omega, \cdot)$ is an arc length parameterization of $\chi(\omega, t)$.

Definition 4.2. A traffic plan $\chi \in \operatorname{TP}_{\mathbb{R}}\left(\mu^{+}, \mu^{-}\right)$is said to be synchronizable (resp. positive synchronizable) if there is some reparameterization $\tilde{\chi} \in \mathrm{TP}_{\mathbb{R}}\left(\mu^{+}, \mu^{-}\right)$(resp. in $\operatorname{TP}\left(\mu^{+}, \mu^{-}\right)$) of $\chi$ such that $\tilde{\chi}$ is synchronized (resp. positive synchronized).

Since $\theta_{\chi}^{\alpha-1} \leq \tilde{\theta}_{\chi}^{\alpha-1}$ with equality if $\chi$ is (positive) synchronized, one can easily deduce that if a traffic plan is synchronized (resp. positive synchronized), then $E_{\mathbb{R}}^{\alpha}(\chi)=C_{\mathbb{R}}^{\alpha}(\chi)\left(\right.$ resp. $\left.E^{\alpha}(\chi)=C^{\alpha}(\chi)\right)$.

The aim of this section is to prove that $E^{\alpha}$-optimal traffic plans are synchronizable. Indeed, optimal traffic plans are such that there is a finite or countable set of points $\left(x_{i}\right)$ and sets $\Omega_{i} \subset \Omega_{x_{i}}$ that form an (almost-) partition of $\Omega$. This fact makes it possible to synchronize independently each tree going through some $x_{i}$, and then harmonize globally these synchronizations thanks to the so-called strict single oriented path property that we now discuss.

The strict single path definition was introduced in [4]. Following these authors, a traffic plan is said to be strict single path if all fibers going through $x$ and $y$ have to coincide between $x$ and $y$. In other terms there is a single path (or none) between any two points of the irrigation network. All optimal traffic plans can then be proven to be strict single path up to the removal of a set of fibers with null measure. For our synchronization purposes, we need to use a slight refinement of this notion, namely what we call the strict single oriented path property. To state this property in precise terms, we first need to introduce some definitions.

Definition 4.3. Let $\chi$ be a loop-free traffic plan, and define $t_{x}(\omega):=\inf \{t: \chi(\omega, t)=x\}$. Let $x, y$ in $S_{\chi}$, and define

$$
\Omega_{\overrightarrow{x y}}:=\left\{\omega \in \Omega_{x}^{\chi} \cap \Omega_{y}^{\chi}: t_{x}(\omega)<t_{y}(\omega)\right\},
$$


the set of fibers passing through $x$ and then through $y$. We denote by $\chi_{x y}$ the restriction of $\chi$ to $\cup_{\omega \in \Omega_{\overrightarrow{x y}}}\{\omega\} \times$ $\left[t_{x}(\omega), t_{y}(\omega)\right]$. It is the traffic plan made of all pieces of fibers of $\chi$ joining $x$ to $y$. Denote its support by $\Gamma^{x y}:=S_{\chi x y}$.

Definition 4.4. A traffic plan $\chi$ has the strict single oriented path property (and we say that $\chi$ is strict single oriented) if, for every pair $x, y$ such that $\left|\Omega_{\overrightarrow{x y}}\right|>0$, all fibers in $\Omega_{\overrightarrow{x y}}$ coincide between $x$ and $y$ with an arc $\Gamma^{x y}$ joining $x$ to $y$, and $\Omega_{\overrightarrow{y x}}=\emptyset$.

By an immediate adaptation of the strict single path property of optimal traffic plans proven in [4], we have the following result.

Proposition 4.5 (strict single oriented path property). Let $\alpha \in[0,1)$ and $\chi$ be an optimal traffic plan such that $E^{\alpha}(\chi)<\infty$. Then, up to removing a zero measure set of fibers, $\chi$ has the strict single oriented path property.

We can now detail the lemmas useful to the prove the synchronizability of $E^{\alpha}$-optima.

Lemma 4.6. If $\chi$ is strict single oriented and $\tilde{\Omega}_{x} \subset \Omega_{x}$, then $\chi_{x}:=\chi\left\llcorner\tilde{\Omega}_{x}\right.$ is synchronizable.

Proof. Let $\tilde{\chi}_{x}(\omega, t)$ be an arc length parameterization of $\chi_{x}(\omega, t)$ such that $\tilde{\chi}_{x}(\omega, 0)=x$. Since $\chi_{x}(\omega, \cdot)$ is injective, there is only one such parameterization. Let us now prove that $\tilde{\chi}_{x}$ is synchronized. Indeed, let us consider a point $y$ in the image of $\chi$. Since $\chi$ is strict single oriented, there is only one path that connects $x$ to $y$ on the support of the traffic plan $\chi_{x}$. This allows to define $l_{\chi_{x}}(y)$ as the distance from $x$ to $y$ through the support of $\chi$. Since $\tilde{\chi}_{x}(\omega, \cdot)$ is parameterized by its arc length, we notice that for all $\omega \in \Omega_{y} \cap \tilde{\Omega}_{x} \tilde{\chi}_{x}\left(\omega, l_{\chi}(y)\right)=y$, i.e. $\tilde{\chi}_{x}$ is synchronized.

Lemma 4.7. Let $\chi_{1}$ and $\chi_{2}$ be synchronized, connected, arc length parameterized, and such that $\chi_{1} \cup \chi_{2}$ is strict single oriented. Then $\chi_{1} \cup \chi_{2}$ is synchronizable.

Proof. If the supports of $\chi_{1}$ and $\chi_{2}$ are disjoint, then $\chi_{1} \cup \chi_{2}$ is already synchronized. Otherwise, let $x$ be a point in the support of both $\chi_{1}$ and $\chi_{2}$. Since $\chi_{1}$ is synchronized, there is some $t_{\chi_{1}}(x)$ such that for all $\omega \in \Omega_{x}^{\chi_{1}}$, $\chi_{1}\left(\omega, t_{\chi_{1}}(x)\right)=x$. We define $t_{\chi_{2}}(x)$ analogously. Let us prove that $t_{\chi_{1}}(x)-t_{\chi_{2}}(x)$ does not depend on the point $x$. Let us consider $x_{1}$ and $x_{2}$ points in the supports of both $\chi_{1}$ and $\chi_{2}$. By connectedness and the strict single oriented path property, there is a unique path on the support of $\chi_{1}$ connecting $x_{1}$ and $x_{2}$ (the same holds for $\left.\chi_{2}\right)$. Since $\chi_{1}$ is arc length parameterized, $t_{\chi_{1}}\left(x_{1}\right)-t_{\chi_{1}}\left(x_{2}\right)$ is exactly the distance from $x_{1}$ to $x_{2}$ through the support of $\chi$ (or its opposite, depending on the orientation of the path). Since $\chi_{1} \cup \chi_{2}$ is strict single oriented, the unique path defined by $\chi_{2}$ is the same as the one of $\chi_{1}$ so that we have:

$$
t_{\chi_{1}}\left(x_{1}\right)-t_{\chi_{1}}\left(x_{2}\right)=t_{\chi_{2}}\left(x_{1}\right)-t_{\chi_{2}}\left(x_{2}\right) .
$$

Thus, shifting the time parameterization of $\chi_{2}$ by $t_{\chi_{1}}(x)-t_{\chi_{2}}(x)$ defines a traffic plan $\tilde{\chi}_{2}$ such that $\chi_{1} \cup \tilde{\chi}_{2}$ is synchronized.

Definition 4.8. We shall say that a traffic plan $\chi$ is finitely (resp. countably) decomposable if there is a finite (resp. countable) set of points $\left(x_{i}\right)$ and sets $\Omega_{i} \subset \Omega_{x_{i}}$ that form a partition of $\Omega$ (almost everywhere).

Proposition 4.9. If $\chi$ is a strict single oriented countably decomposable traffic plan, then it is synchronizable.

Proof. Let $\Omega_{i} \subset \Omega_{x_{i}}$ defining a countable decomposition of $\chi$, and let us denote $\chi_{i}:=\chi\left\llcorner\Omega_{i}\right.$. Lemma 4.6 ensures that all the $\chi_{i}$ are synchronizable, and we denote by $\tilde{\chi}_{i}$ an equivalent synchronized traffic plans. Since $\chi$ is strict single oriented, $\cup_{i} \tilde{\chi}_{i}$ is strict single oriented. Thus, by induction, the repeated application of Lemma 4.7 allows to define a synchronized traffic plan $\tilde{\chi}$ that is the union of time shifted versions of $\tilde{\chi}_{i}$. Such a traffic plan $\tilde{\chi}$ is a time-reparameterization of $\chi$ that is synchronized.

Proposition 4.10. If $\mu^{+}$is finite atomic, then any optimal traffic plan $\chi \in \operatorname{TP}_{\mathbb{R}}\left(\mu^{+}, \mu^{-}\right)$is positive synchronizable. 
Proof. Let $\left(x_{i}\right)_{i=1}^{n}$ be a finite sequence such that $\mu^{+}:=\sum_{i=1}^{n} a_{i} \delta_{x_{i}}$. The sets defined by $\Omega_{1}:=\Omega_{x_{1}}$ and $\Omega_{i}:=\Omega_{x_{i}} \backslash\left(\cup_{j<i} \Omega_{j}\right)$ for $i>1$, yield a partition of $\Omega$, so that $\chi$ is finitely decomposable. Since $\chi$ is optimal, it is strict single oriented, and Proposition 4.9 ensures that $\chi$ is synchronizable. Since $\mu^{+}$is atomic, by the construction of the reparameterization given in Lemma 4.7 it is simple to see that, with a suitable time shifting, $\chi$ is also positive synchronizable.

Proposition 4.11. Any optimal traffic plan $\chi \in \mathrm{TP}_{\mathbb{R}}$ is synchronizable.

Proof. Any optimal traffic plan is countably decomposable (see [4], Lem. 3.11) and strict single oriented. Thus, by Proposition 4.9, it is synchronizable.

\section{Equivalence of the DyNAMiCAL AND ClASSiCAL IRRIGATION PROBLEMS}

In the same way as for $E^{\alpha}$, we define:

$$
C^{\alpha}\left(\mu^{+}, \mu^{-}\right):=\inf _{\operatorname{TP}\left(\mu^{+}, \mu^{-}\right)} C^{\alpha}(\chi), \quad C_{\mathbb{R}}^{\alpha}\left(\mu^{+}, \mu^{-}\right):=\inf _{\operatorname{TP}_{\mathbb{R}}\left(\mu^{+}, \mu^{-}\right)} C_{\mathbb{R}}^{\alpha}(\chi) .
$$

Theorem 5.1. If $\mu^{+}$is finite atomic, then, for all $\alpha \in[0,1]$,

$$
E^{\alpha}\left(\mu^{+}, \mu^{-}\right)=C^{\alpha}\left(\mu^{+}, \mu^{-}\right),
$$

and

$$
E_{\mathbb{R}}^{\alpha}\left(\mu^{+}, \mu^{-}\right)=C_{\mathbb{R}}^{\alpha}\left(\mu^{+}, \mu^{-}\right) .
$$

Proof. We remark that, by the definition of $E^{\alpha}$ and $C^{\alpha}$, we immediately have the inequality

$$
E^{\alpha}(\chi) \leq C^{\alpha}(\chi) \text { for all traffic plan } \chi
$$

so that,

$$
E^{\alpha}\left(\mu^{+}, \mu^{-}\right) \leq C^{\alpha}\left(\mu^{+}, \mu^{-}\right) \quad \forall \alpha \in[0,1] .
$$

Let $\chi$ be a minimizer of $E^{\alpha}$. Proposition 4.10 ensures that there is a reparameterization $\tilde{\chi}$ of $\chi$ such that $\tilde{\chi}$ is positive synchronized, so that

$$
E^{\alpha}\left(\mu^{+}, \mu^{-}\right)=E^{\alpha}(\chi)=E^{\alpha}(\tilde{\chi})=C^{\alpha}(\tilde{\chi}) .
$$

Thus, $E^{\alpha}\left(\mu^{+}, \mu^{-}\right)=C^{\alpha}\left(\mu^{+}, \mu^{-}\right)$for all $\alpha \in[0,1]$. Finally, Proposition 4.11 yields $E_{\mathbb{R}}^{\alpha}\left(\mu^{+}, \mu^{-}\right)=C_{\mathbb{R}}^{\alpha}\left(\mu^{+}, \mu^{-}\right)$ for all $\alpha \in[0,1]$.

By Proposition 4.11, we also have:

Theorem 5.2. Let $\mu^{+}$and $\mu^{-}$be two probability measures. Then

$$
E_{\mathbb{R}}^{\alpha}\left(\mu^{+}, \mu^{-}\right)=C_{\mathbb{R}}^{\alpha}\left(\mu^{+}, \mu^{-}\right) .
$$

Theorem 5.2 states the equivalence of the cost given by the dynamical and the classical irrigation problem. Concerning minimizers, we can observe as a direct consequence of Theorem 5.2 and (5.1) that every $C_{\mathbb{R}}^{\alpha}$-minimizer is an $E_{\mathbb{R}}^{\alpha}$-minimizer. Conversely, by Proposition 4.11 , any $E_{\mathbb{R}}^{\alpha}$-minimizer can be reparameterized so that it gives a $C_{\mathbb{R}}^{\alpha}$-minimizer. The same considerations are true for $E^{\alpha}$ and $C^{\alpha}$ if $\mu^{+}$is finite atomic thanks to Proposition 4.10. Thus, in both these cases, the extended dynamical and classical irrigation problems yield exactly the same minimizers (up to reparameterization). In particular, as a by-product, we obtain the existence of $C^{\alpha}$-minimizers if $\mu^{+}$is finite atomic, and existence of $C_{\mathbb{R}}^{\alpha}$-minimizers in general.

As a particular consequence of the fact that every $C_{\mathbb{R}}^{\alpha}$-minimizer is an $E_{\mathbb{R}}^{\alpha}$-minimizer, we notice that $C_{\mathbb{R}}^{\alpha}$-minimizers inherit all the regularity properties of $E_{\mathbb{R}}^{\alpha}$-minimizers (the same holds for $C^{\alpha}$-minimizers, in the case $\mu^{+}$is finite atomic). Thus we can translate the regularity results in Section 2 in the $C_{\mathbb{R}}^{\alpha}$ framework. 
Proposition 5.3. Let $\alpha \in[0,1], \mu^{+}$and $\mu^{-}$be finite atomic measures, and $\chi \in \operatorname{TP}\left(\mu^{+}, \mu^{-}\right)$be a $C^{\alpha}$-minimizer. Then $\chi$ is a finite tree made of segments.

Theorem 5.4. Let $\alpha \in\left(1-\frac{1}{N}, 1\right), \mu^{+}$and $\mu^{-}$be probability measures, and $\chi \in \operatorname{TP}_{\mathbb{R}}\left(\mu^{+}, \mu^{-}\right)$be a $C_{\mathbb{R}}^{\alpha}$-minimizer. Assume that the supports of $\mu^{+}$and $\mu^{-}$are at positive distance. In any closed ball $B(x, r)$ not meeting the supports of $\mu^{+}$and $\mu^{-}$, the traffic plan $\chi$ has the structure of a finite graph.

\section{Stability with Respect to the Cost}

In this section we study the regularity with respect to $\alpha$ of $E^{\alpha}\left(\mu^{+}, \mu^{-}\right)$for fixed $\mu^{+}$and $\mu^{-}$. By the equivalence of $E_{\mathbb{R}}^{\alpha}$ and $C_{\mathbb{R}}^{\alpha}$ (Th. 5.2), and $E^{\alpha}$ and $C^{\alpha}$ when $\mu^{+}$is finite atomic (Th. 5.1), one can deduce similar stability results for the dynamical cost.

We start studying the regularity with respect to $\alpha$ of $E^{\alpha}(\chi)$ for a fixed traffic plan $\chi$.

Lemma 6.1. Let $\chi$ be a traffic plan. Then $[0,1] \ni \alpha \mapsto E^{\alpha}(\chi) \in \mathbb{R}^{+} \cup\{+\infty\}$ is non-increasing. Fix now $\alpha \in[0,1)$. Then:

(i) If $E^{\alpha}(\chi)<+\infty$, then $\beta \mapsto E^{\beta}(\chi)$ is finite and continuous on $[\alpha, 1]$.

(ii) If $E^{\alpha}(\chi)=+\infty$, then $E^{\alpha_{n}}(\chi) \rightarrow+\infty$ for any decreasing sequence $\alpha_{n} \searrow \alpha$.

Proof. The monotonicity of $\alpha \mapsto E^{\alpha}(\chi)$ is trivial.

Let $\chi$ be such that $E^{\alpha}(\chi)<+\infty$ and let $\beta_{n} \in[\alpha, 1]$ such that $\beta_{n} \rightarrow \beta$. For all $(\omega, t) \in \Omega \times \mathbb{R}^{+}$, we have

$$
\theta_{\chi}(\chi(\omega, t))^{\beta_{n}-1} \rightarrow \theta_{\chi}(\chi(\omega, t))^{\beta-1} .
$$

In addition, as $\theta_{\chi}(\chi(\omega, t)) \leq 1$, we have

$$
0 \leq \theta_{\chi}(\chi(\omega, t))^{\beta_{n}-1} \leq \theta_{\chi}(\chi(\omega, t))^{\alpha-1}
$$

Thus, since

$$
E^{\alpha}(\chi)=\int_{\Omega} \int_{\mathbb{R}^{+}} \theta_{\chi}(\chi(\omega, t))^{\alpha-1}|\dot{\chi}(\omega, t)| \mathrm{d} t \mathrm{~d} \omega<\infty,
$$

the dominated convergence theorem ensures the convergence of $E^{\beta_{n}}(\chi)$ to $E^{\beta}(\chi)$.

Let us now consider a traffic plan $\chi$ such that $E^{\alpha}(\chi)=+\infty$, and let $\alpha_{n}$ be a decreasing sequence converging to $\alpha$. Then for all $(\omega, t) \in \Omega \times \mathbb{R}^{+}, \theta_{\chi}(\chi(\omega, t))^{\alpha_{n}-1}$ is increasingly converging to $\theta_{\chi}(\chi(\omega, t))^{\alpha-1}$. Thus, the monotone convergence theorem ensures that $E^{\alpha_{n}}(\chi) \rightarrow+\infty$.

Now we can study the stability of $E^{\alpha}\left(\mu^{+}, \mu^{-}\right)$with respect to $\alpha$.

Proposition 6.2. Let $\mu^{+}$and $\mu^{-}$be two probability measures. The function $[0,1] \ni \alpha \mapsto E^{\alpha}\left(\mu^{+}, \mu^{-}\right) \in$ $\mathbb{R}^{+} \cup\{+\infty\}$ is non-increasing, right continuous and left lower semicontinuous.

Proof. For simplicity of notation set $f(\alpha):=E^{\alpha}\left(\mu^{+}, \mu^{-}\right)$. Observe that, since $\alpha \mapsto E^{\alpha}(\chi)$ is non-increasing for all $\chi, f$ is non-increasing being an infimum of non-increasing functions. Thus, $f$ is left lower semicontinuous, i.e.

$$
\liminf _{n} f\left(\alpha_{n}\right) \geq f(\alpha) \text { for all } \alpha_{n} \nearrow \alpha
$$

In what follows, $\chi_{\beta}$ will always denote an optimal traffic plan for the exponent $\beta$, i.e. such that $E^{\beta}\left(\chi_{\beta}\right)=f(\beta)$. Let us consider a decreasing sequence $\alpha_{n}$ such that $\alpha_{n} \searrow \alpha$ and a sequence of optimal traffic plans $\chi_{\alpha_{n}}$.

By Lemma 6.1 and the optimality of $\chi_{\alpha_{n}}$ for $E^{\alpha_{n}}$ we get

$$
f(\alpha)=E^{\alpha}\left(\chi_{\alpha}\right)=\lim _{n} E^{\alpha_{n}}\left(\chi_{\alpha}\right) \geq \limsup _{n} E^{\alpha_{n}}\left(\chi_{\alpha_{n}}\right) \geq \liminf _{n} E^{\alpha_{n}}\left(\chi_{\alpha_{n}}\right) .
$$


If $\lim \inf _{n} E^{\alpha_{n}}\left(\chi_{\alpha_{n}}\right)=+\infty$, there is nothing to prove. Otherwise, up to apply the reparameterization argument used to prove Corollary 2.9, we can assume that $\chi_{\alpha_{n}} \in \mathrm{TP}_{C}$ for some $C>0$. Thus, by Proposition 2.8, there is a subsequence $\chi_{\alpha_{n_{k}}}$ such that

$$
\chi_{\alpha_{n_{k}}} \rightarrow \chi \quad \text { and } \quad \liminf _{k} E^{\alpha_{n_{k}}}\left(\chi_{\alpha_{n_{k}}}\right)=\liminf _{n} E^{\alpha_{n}}\left(\chi_{\alpha_{n}}\right)
$$

Recalling that $\alpha \mapsto E^{\alpha}(\chi)$ is non-increasing, and that $E^{\alpha_{m}}$ is lower semicontinuous for $m$ fixed, we have

$$
\liminf _{k} E^{\alpha_{n_{k}}}\left(\chi_{\alpha_{n_{k}}}\right) \geq \liminf _{k} E^{\alpha_{m}}\left(\chi_{\alpha_{n_{k}}}\right) \geq E^{\alpha_{m}}(\chi) \text { for all } m
$$

By Lemma 6.1, $\lim _{m} E^{\alpha_{m}}(\chi)=E^{\alpha}(\chi)$ so that (6.1), (6.2) and (6.3) yield

$$
f(\alpha) \geq \limsup _{n} f\left(\alpha_{n}\right) \geq \liminf _{n} f\left(\alpha_{n}\right) \geq E^{\alpha}(\chi) \geq f(\alpha) .
$$

Corollary 6.3. Let $\alpha_{n} \in[0,1]$ be a decreasing sequence converging to $\alpha$, and let $\mu^{+}$and $\mu^{-}$be two probability measures. If $\chi_{\alpha_{n}}$ are optimal traffic plans for $E^{\alpha_{n}}$ and $\chi_{\alpha_{n}} \rightarrow \chi$, then $\chi$ is optimal for $E^{\alpha}$.

Proof. By Proposition 6.2, and since $\alpha \mapsto E^{\alpha}(\chi)$ is non-increasing and $E^{\alpha_{m}}$ is lower semicontinuous for fixed $m$, we have

$$
E^{\alpha}\left(\mu^{+}, \mu^{-}\right)=\lim _{n} E^{\alpha_{n}}\left(\chi_{\alpha_{n}}\right) \geq \liminf _{n} E^{\alpha_{m}}\left(\chi_{\alpha_{n}}\right) \geq E^{\alpha_{m}}(\chi) .
$$

Since by Lemma $6.1 \lim _{m} E^{\alpha_{m}}(\chi)=E^{\alpha}(\chi), \chi$ is optimal.

If we now constrain $\alpha$ to be in $\left(1-\frac{1}{N}, 1\right]$, we are able to say more. Indeed, in this case, Proposition 2.14 allows us to approximate $\mu^{+}$and $\mu^{-}$with atomic measures $\mu_{n}^{+}$and $\mu_{n}^{-}$in such a way that $E^{\alpha}\left(\mu^{+}, \mu^{-}\right)$is a uniform limit (locally in $\alpha$ ) of $E^{\alpha}\left(\mu_{n}^{+}, \mu_{n}^{-}\right)$. Then it is sufficient to prove that $E^{\alpha}\left(\mu_{n}^{+}, \mu_{n}^{-}\right)$is continuous for any $n$, in order to have that $E^{\alpha}\left(\mu^{+}, \mu^{-}\right)$is continuous on $\left(1-\frac{1}{N}, 1\right]$.

Lemma 6.4. Let $\mu^{+}=\sum_{i=1}^{k_{1}} a_{i} \delta_{x_{i}}$ and $\mu^{-}=\sum_{i=1}^{k_{2}} b_{i} \delta_{y_{i}}$ be atomic measures such that $\sum_{i=1}^{k_{1}} a_{i}=\sum_{i=1}^{k_{2}} b_{i}$ (the irrigating and the irrigated measure have the same mass). Then $\alpha \mapsto E^{\alpha}\left(\mu^{+}, \mu^{-}\right)$is continuous on $[0,1]$.

Proof. By Proposition 2.17, we know that, for all $\alpha \in[0,1]$, an optimum for the irrigation problem can be viewed as a weighted and oriented finite graph $G$. Then, if we call $\chi_{\alpha}$ an optimum for $E^{\alpha}$, we have

$$
E^{\alpha}\left(\mu^{+}, \mu^{-}\right)=E^{\alpha}\left(\chi_{\alpha}\right)=\sum_{i=1}^{n_{\alpha}} l_{i} m_{i}^{\alpha}
$$

where the $l_{i}$ and $m_{i}$ are respectively the lengths and weights of the edges of $G$. Then, since

$$
\beta \mapsto E^{\beta}\left(\chi_{\alpha}\right)=\sum_{i=1}^{n_{\alpha}} l_{i} m_{i}^{\beta}
$$

is continuous and finite on $[0,1]$, we see that $E^{\alpha}\left(\mu^{+}, \mu^{-}\right)$is finite on $[0,1]$. Moreover we already know by Proposition 6.2 that $E^{\alpha}\left(\mu^{+}, \mu^{-}\right)$is left lower semicontinuous and right continuous. So, in order to conclude it is sufficient to prove that $E^{\alpha}\left(\mu^{+}, \mu^{-}\right)$is left upper semicontinuous. Let $\left(\alpha_{n}\right)$ be a sequence such that $\alpha_{n} \nearrow \alpha$. The continuity of $\beta \mapsto E^{\beta}\left(\chi_{\alpha}\right)$ ensures that

$$
\underset{n}{\limsup } E^{\alpha_{n}}\left(\mu^{+}, \mu^{-}\right)=\underset{n}{\limsup } E^{\alpha_{n}}\left(\chi_{\alpha_{n}}\right) \leq \limsup _{n} E^{\alpha_{n}}\left(\chi_{\alpha}\right)=E^{\alpha}\left(\chi_{\alpha}\right)=E^{\alpha}\left(\mu^{+}, \mu^{-}\right)
$$

Theorem 6.5. Let $\alpha_{n} \in\left[1-\frac{1}{N}, 1\right]$ be a sequence converging to $\alpha$. If the traffic plans $\chi_{\alpha_{n}}$ are optimal for $E^{\alpha_{n}}$ and $\chi_{\alpha_{n}} \rightarrow \chi$, then $\chi$ is optimal for $E^{\alpha}$. 
Proof. By Proposition 2.14, for all $\mu^{+}$and $\mu^{-}$there are atomic measures $\mu_{n}^{+}$and $\mu_{n}^{-}$such that $E^{\alpha}\left(\mu_{n}^{+}, \mu_{n}^{-}\right)$ converges uniformly to $E^{\alpha}\left(\mu^{+}, \mu^{-}\right)$on $\left(1-\frac{1}{N}, 1\right]$. Lemma 6.4 asserts that $\alpha \mapsto E^{\alpha}\left(\mu_{n}^{+}, \mu_{n}^{-}\right)$is continuous, so that $\alpha \mapsto E^{\alpha}\left(\mu^{+}, \mu^{-}\right)$is continuous on $\left(1-\frac{1}{N}, 1\right]$. By the same kind of argument as in the proof of Corollary 6.3, we deduce that $\chi$ is optimal. If $\alpha=1-\frac{1}{N}$, we can suppose that up to a subsequence $\alpha_{n} \searrow \alpha$, so that Corollary 6.3 ensures that $\chi$ is optimal (possibly trivially optimal in the case $E^{\alpha}(\chi)=\infty$ ).

Remark 6.6. In the case $\alpha=1$, the irrigation problem for the cost $E^{\alpha}$ is equivalent to the classical MongeKantorovich problem (see $[9,11,14]$ ). For that particular case, Theorem 6.5 ensures that the transference plan associated to a sequence of optimal traffic plans $\chi_{\alpha_{n}}$, where $\alpha_{n} \rightarrow 1$, converges, up to a subsequence, to an optimal transference plan for the Monge-Kantorovich problem.

Acknowledgements. We warmly thank Patrick Bernard and Filippo Santambrogio for useful comments and remarks. The second author gratefully acknowledges the hospitality of the École Normale Supérieure of Lyon, where this paper was written.

\section{REFERENCES}

[1] L. Ambrosio, N. Fusco and D. Pallara, Functions of bounded variations and free discontinuity problems, Oxford Mathematical Monographs. The Clarendon Press, Oxford University Press (2000).

[2] M. Bernot, Irrigation and Optimal Transport. Ph.D. thesis, École Normale Supérieure de Cachan, France (2005). Available at http://www. umpa.ens-lyon.fr/ mbernot.

[3] M. Bernot, V. Caselles and J.-M. Morel, Traffic plans. Publ. Mat. 49 (2005) 417-451.

[4] M. Bernot, V. Caselles and J.-M. Morel, The structure of branched transportation networks. Calc. Var. Partial Differential Equations (online first). DOI: 10.1007/s00526-007-0139-0.

[5] A. Brancolini, G. Buttazzo and F. Santambrogio, Path functionals over Wasserstein spaces. J. EMS 8 (2006) $414-434$.

[6] W. D'Arcy Thompson, On Growth and Form. Cambridge University Press (1942).

[7] R.M. Dudley, Real Analysis and Probability. Cambridge University Press (2002).

[8] E.N. Gilbert, Minimum cost communication networks. Bell System Tech. J. 46 (1967) 2209-2227.

[9] L. Kantorovich, On the transfer of masses. Dokl. Acad. Nauk. USSR 37 (1942) 7-8.

[10] F. Maddalena, S. Solimini and J.M. Morel, A variational model of irrigation patterns. Interfaces and Free Boundaries 5 (2003) 391-416.

[11] G. Monge, Mémoire sur la théorie des déblais et de remblais. Histoire de l'Académie Royale des Sciences de Paris (1781) 666-704.

[12] J.D. Murray, Mathematical Biology, Biomathematics Texts 19. Springer (1993).

[13] A.M. Turing, The chemical basis of morphogenesis. Phil. Trans. Soc. Lond. B237 (1952) 37-72.

[14] C. Villani, Topics in optimal transportation, Graduate Studies in Mathematics 58. American Mathematical Society, Providence, RI (2003).

[15] Q. Xia, Optimal paths related to transport problems. Commun. Contemp. Math. 5 (2003) 251-279. 